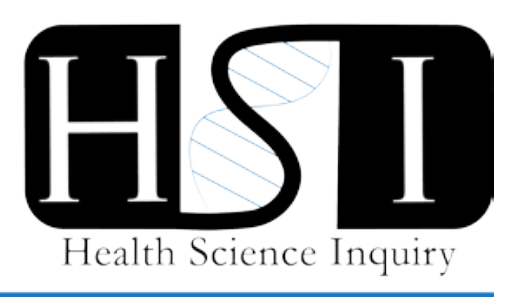

\title{
Waiting in transition: access to rehabilitation care for adults with chronic neurological conditions in Ontario
}

\author{
Shikha Gupta \\ School of Rehabilitation Therapy, Queen's University
}

\section{Top Submission}

\section{Chronic neurological conditions in Canada and rehabilitation care}

One of the leading causes of chronic disability in the Canadian population is neurological conditions ${ }^{1}$ that affect an estimated population of 3.6 million in the community and 170,000 in long-term institutions [1]. Evidence has been established that rehabilitation interventions are effective for functional recovery after neurological trauma, illness or injury [2]. Fundamental to neurological rehabilitation is the appreciation that different patients need systematic care at different stages in their recovery [3]. Systematic care means the adequate amount, intensity and volume of care that is required at the right time to achieve individual health outcomes at every stage in the rehabilitation process. For systematic care, services need to be designed and delivered in such a way to ensure continuity of care for the patients, from hospital to home and then to the community. However, literature highlights that many people with chronic neurological conditions do not receive the right services at the right time and wait at every step in transition [4].

\section{Factors affecting timely access to rehabilitation care}

Critical analysis of available literature suggests that there are many factors that play a role at each of the stages of the rehabilitation care pathway [5]. During acute and subacute phases, individual's clinical characteristics, especially the motor and cognitive status assessed by functional independence measurement scores, determine whether the patient will receive in-patient rehabilitation or not [6]. However, within inpatient rehabilitation the focus remains on early discharge, given the current policy emphasis on cost shifting to the community and a belief that community-based rehabilitation ensures better recovery [7]. This means that as soon as the patient reaches the community stage, access to rehabilitation care becomes severely restricted as a result of limited resources and policy decisions resulting in restructuring of the current health system (Figure 1).

The growth of the 65+ age cohort and increasing rates of chronic conditions has increased the demand for rehabilitation services in Ontario. Despite increasing demand, the Ontario government adopted several structural measures directed towards cost-containment and privatization [7]. Following this restructuring, only partial coverage for rehabilitation services was provided based on eligibility criteria ${ }^{2}$. As a result, individuals who do not meet eligibility have to obtain services available privately or limited by the home-care coordinating agencies [8]. Even for the individuals who meet eligibility criteria, there is capping on the services which means that limited services are provided based on predefined amounts of hours per service. As a result, volume of services provided to the clients are capped with the potential for clients to purchase additional services from provider agencies. Concurrent to this, numbers for therapist-population ratios are concerning. An estimated ratio of therapists to the population is 51 for physiotherapists (PTs) and 36 for occupational therapists (OTs) out of 100,000 in Ontario [9]. Considering that there are more than 5,600 people with neurological conditions per 100,000 people in Ontario, these numbers for PTs and OTs available will further shrink simultaneous to an aging population (Table 1).

\footnotetext{
${ }^{1}$ The 11 most common neurological conditions in Canada are Alzheimer's disease, amyotrophic lateral sclerosis, brain tumors, cerebral palsy, epilepsy, head injury, headaches, multiple sclerosis, Parkinson's disease, spinal injuries and stroke.

${ }^{2}$ Eligibility criteria include: (1) residents aged over 65 or under 20 years, (2) those receiving social support through the Ontario Disability Support Program, Family Benefits or Ontario Works, (3) residents of long-term care facilities and (4) those who are returned to the community following hospital discharge.
} 


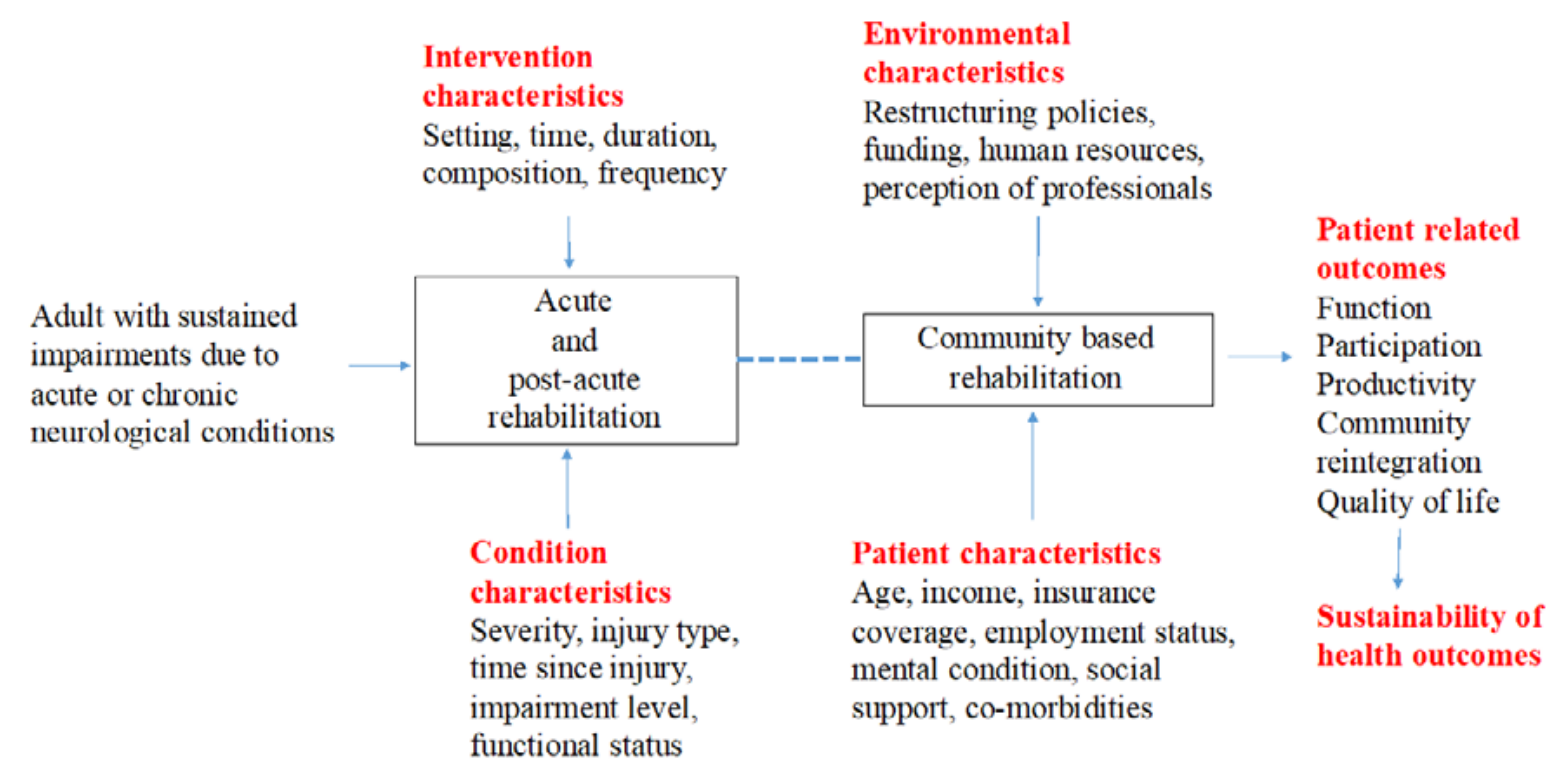

Figure 1: Factors affecting access to timely rehabilitation services across rehabilitation care pathways

\section{Consequences of wait times for rehabilitation care}

Withholding necessary care from someone who is in need of services can have unwanted health implications for the person and lead to negative medical outcomes. Consequences of wait times can be serious and grave, especially for adults with neurological conditions, including prolonged suffering from pain, discomfort, loss of function and mental agony [10]. Although in recent years, much attention has been given to limiting waiting times in healthcare, the majority of these initiatives have addressed medical and surgical procedures such as cardiac surgeries, knee replacements and chemotherapy. Home based or community based rehabilitation care has not been considered as medically necessary or something that needs to be provided urgently. Even in the settings where triage, a mechanism of referral prioritization by clinicians, is practiced for rehabilitation services, there is no guarantee of timely access based on need [4].

\section{Conclusion and future directions}

It is apparent that adults with neurological conditions have to wait at every step in transition during rehabilitation, from entry to exit from the system, and both time (i.e. limited amounts of hours per service) and timing (i.e. delay) of rehabilitation services affect their trajectory of recovery. This calls into question the appropriateness of the system's response to the rehabilitation care needs of adults living with chronic neurological conditions. Some of the gaps that have been identified in the literature indicate the need to understand how timeliness of care influence the

Table 1: Estimated therapist-population ratio in Canada and Ontario

\begin{tabular}{|c|c|c|}
\hline Estimates & Canada & Ontario \\
\hline Total Population (2014) & $35,544,600$ & $13,685,200$ \\
\hline Population with neurological conditions (2015) & $3,870,000$ & 770,259 \\
\hline Population with neurological conditions per 100,000 population & 10,887 & 5,600 \\
\hline Total number of PTs (2013) & 19,253 & 6950 \\
\hline Total number of OTs (2013) & 14,351 & 4892 \\
\hline Number of PTs per 100,000 population (2013) & 55 & 51 \\
\hline Number of OTs per 100,000 population (2013) & 41 & 36 \\
\hline
\end{tabular}


patterns of recovery and health outcomes of patients with neurological conditions. Longitudinal research is required to address this gap as understanding these processes will contribute in framing effective systems that ensure timely access to services at all points of transition in the healthcare system.

\section{References}

[1] Kirkland, J. L., Tchkonia, T., Zhu, Y., Niedernhofer, L. J., Robbins, P. D. (2017) The Clinical Potential of Senolytic Drugs. J Am Geriatr Soc, 65 (10), 22972301. Available from: http://doi.wiley.com/10.1111/jgs.14969.

[2] Muñoz-Espín, D., Serrano, M. (2014) Cellular senescence: from physiology to pathology. Nat Rev Mol Cell Biol, 15 (7), 482496. Available from: http://www.nature.com/articles/nrm3823.

[3] Palmer, A. K., Tchkonia, T., LeBrasseur, N. K., Chini, E.N., Xu, M., Kirkland, J. L (2015) Cellular Senescence in Type 2 Diabetes: A Therapeutic Opportunity. Diabetes, 64 (7), 22892298. Available from: http://www.ncbi.nlm.nih.gov/pubmed/26106186.

[4] Lecot, P., Alimirah, F., Desprez, P-Y., Campisi, J., Wiley, C. (2016) Context-dependent effects of cellular senescence in cancer development. $\mathrm{Br} J$ Cancer, 114 (11):11801184. Available from: http://www.nature.com/articles/bjc2016115.

[5] Coppé, J-P., Desprez, P-Y., Krtolica, A., Campisi, J. (2010) The Senescence-Associated Secretory Phenotype: The Dark Side of Tumor Suppression. Annu Rev Pathol Mech Dis, 5 (1), 99118. Available from: http://www.annualreviews.org/doi/10.1146/annurevpathol-121808-102144.

[6] Gregor, M. F., Hotamisligil, G. S. (2011) Inflammatory Mechanisms in Obesity. Annu Rev Immunol, 29 (1), 415445. Available from: http://www.annualreviews.org/doi/10.1146/annurevimmunol-031210-101322.

[7] Minamino, T., Orimo, M., Shimizu, I., Kunieda, T., Yokoyama, M., Ito, T., et al. (2009) A crucial role for adipose tissue p53 in the regulation of insulin resistance. Nat Med, 15 (9), 10821087. Available from: http://www.nature.com/articles/nm.2014.

[8] Sone, H., Kagawa, Y. (2005) Pancreatic beta cell senescence contributes to the pathogenesis of type 2 diabetes in high-fat diet-induced diabetic mice. Diabetologia, 48 (1), 5867. Available from: http://link.springer.com/10.1007/s00125-004-1605-2.

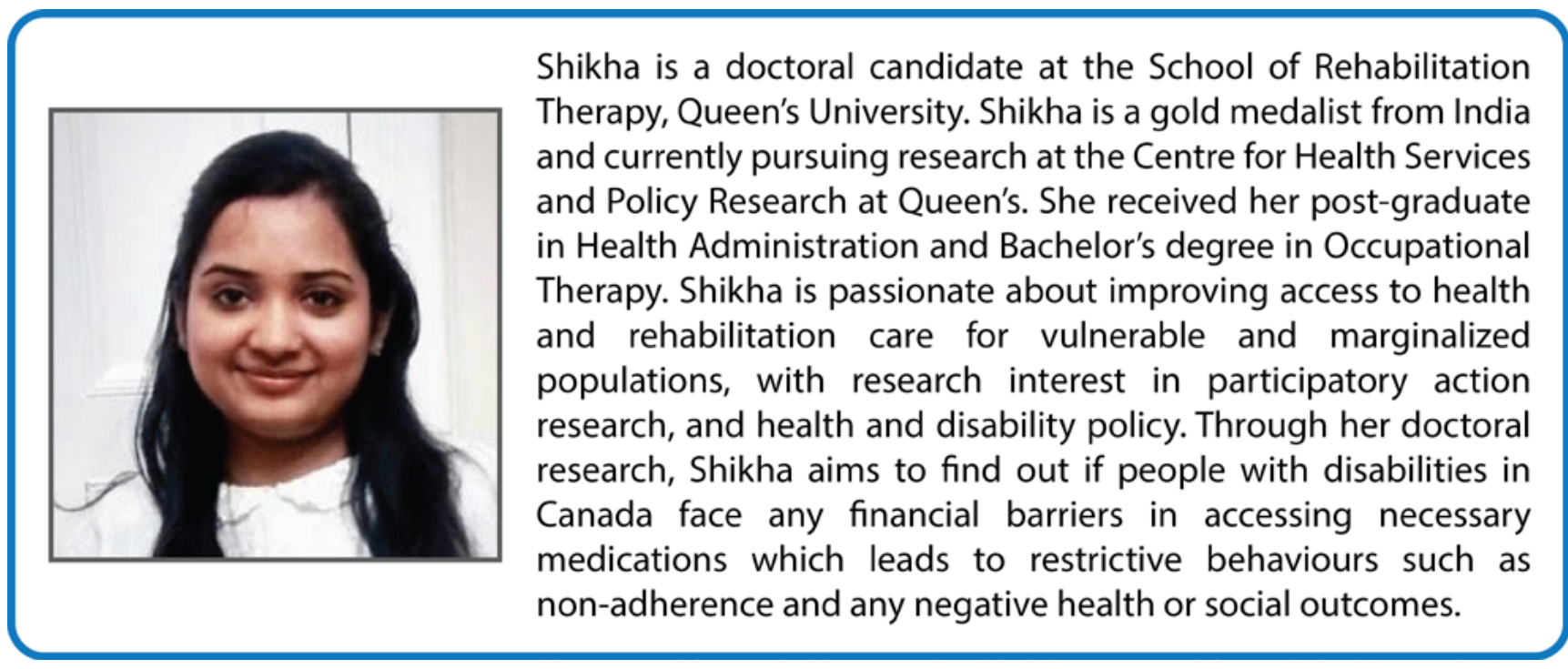

